\title{
Convergent relaxations of polynomial matrix inequalities and static output feedback
}

\author{
Didier Henrion, Jean-Bernard Lasserre
}

\begin{abstract}
Using a moment interpretation of recent results on sum-of-squares decompositions of non-negative polynomial matrices, we propose a hierarchy of convex linear matrix inequality (LMI) relaxations to solve non-convex polynomial matrix inequality (PMI) optimization problems, including bilinear matrix inequality (BMI) problems. This hierarchy of LMI relaxations generates a monotone sequence of lower bounds that converges to the global optimum. Results from the theory of moments are used to detect whether the global optimum is reached at a given LMI relaxation, and if so, to extract global minimizers that satisfy the PMI. The approach is successfully applied to PMIs arising from static output feedback design problems.
\end{abstract}

Index Terms-Polynomial matrix, nonconvex optimization, convex optimization, static output feedback design

\section{INTRODUCTION}

Most of synthesis problems for linear systems can be formulated as polynomial matrix inequality (PMI) optimization problems in the controller parameters, a particular case of which are bilinear matrix inequalities (BMI) [7]. Generally, these PMI problems are non-convex and hence, difficult to solve. Only in very specific cases (static state feedback, dynamic output feedback controller of the same order as the plant) suitable changes of variables or subspace projections have been found to convexify the design problem and derive equivalent linear matrix inequality (LMI) formulations [2], [27], [26]. However, for several basic control problems such as PID design, simultaneous stabilization or static output feedback design, no equivalent convex LMI formulation is known. As a consequence, solving PMI problems is a difficult numerical challenge, and there is still a lack of efficient computer-aided control system design (CACSD) algorithms to address them satisfactorily.

Traditionally, non-convex PMI optimization problems can be tackled either locally or globally:

- Local methods can be highly sensitive to the choice of the initial point, and generally provide a guarantee of convergence to points satisfying necessary first order optimality conditions only. Several local methods have been reported in the technical literature, but up to our knowledge, the first and so far only publicly available implementation of a BMI solver is PENBMI [16], [17], based on a penalty function and augmented Lagrangian algorithm;

D. Henrion and J. B. Lasserre are with LAAS-CNRS, 7 Avenue du Colonel Roche, 31077 Toulouse, France.

D. Henrion is also with the Department of Control Engineering, Faculty of Electrical Engineering, Czech Technical University in Prague, Technická 2, 16627 Prague, Czech Republic.

J.-B. Lasserre is also with the Mathematics Institute of Toulouse, Paul Sabatier University, 31602 Toulouse, France.
- Global methods, based on branch-and-bound schemes and alike [6], are generally highly demanding computationally. Efficient LMI bounding strategies can be designed to derive tight upper and lower bounds on non-convex objective functions and feasible sets [4], [5], but one can hardly avoid the combinatorial explosion inherent to branching schemes. Consequently these global methods are restricted to small (if not academic) problem instances only.

In this paper, we propose another strategy to overcome the above shortcomings of local and global methods.

- On the one hand, our method is global in the sense that it solves PMI problems and when finite convergence occurs, it also provides a numerical certificate of global optimality (several distinct global optima can be found without any combinatorial branching strategy).

- On the other hand, our method uses the LMI formalism and makes extensive use of convex semidefinite programming (SDP). In particular, we only rely on efficient SDP codes already available, which avoids the considerably difficult work of developing a specific algorithm and solver.

The main idea behind the PMI optimization method described in this paper is along the lines of that developed in [19] for scalar polynomial constraints. Based on the theory of sum-of-squares positive polynomials and its dual theory of moments, a hierarchy of LMI relaxations of increasing dimensions is built up in such a way that the designer has to trade off between the expected accuracy and the computational load, with the theoretical guarantee of asymptotic convergence to the global optimum. Moreover, and as finite convergence typically occurs in many cases, numerical linear algebra procedures are available to detect global optimality and extract global optimizers. Practice reveals that for small to medium global optimization problems, and up to machine precision, finite convergence eventually occurs, that is, the global optimum is reached at some LMI relaxation of reasonable dimension. See [9] for a description of a Matlab implementation with an extensive set of numerical examples, and [11] for applications in systems control.

Interestingly enough, the feasible set of any PMI problem is a semi-algebraic set and can be also represented by finitely many polynomial scalar inequalities. However, typical in this latter scalar representation is a high degree occurring for at least one polynomial, which makes the scalar approach [19] impractical in view of the present status of SDP solvers.

Our contribution is to extend the scalar moment approach of [19] to the matrix case, using recent results by Hol and 
Scherer [14], [15] and Kojima [18] on sum-of-squares of polynomial matrices, and deriving a dual theory of moments. Thanks to the dual interpretation provided by the theory of moments, we can certify global optimality and extract the optimizers. In many applications this point is crucial, as one is primarily interested in finding a global minimizer, rather than just the global optimum. In other words, using standard terminology in optimization, we can say that our method is primal as it works in a lifted primal space (the moments) of the original primal space, whereas papers [14], [15], [18] describe a dual approach which yields the optimal value but not the minimizers, exactly as Lagrangian relaxation methods in optimization yield optimal Lagrange multipliers but not minimizers.

The outline of the paper is as follows. In Section II we provide the matrix analogues of moment and localizing matrices defined in [19] for the scalar case, and a specific test to detect global optimality at a given LMI relaxation. In Section III, we apply this methodology to solve PMI problems coming from static output feedback (SOF) design problems. A salient feature of our approach is the particular algebraic (or polynomial) formulation of the SOF. Indeed, in contrast with the standard state-space BMI approach that introduces a significant number of instrumental additional Lyapunov variables, the only decision variables of our SOF PMI problem are precisely the entries of the feedback gain matrix.

\section{LMI RELAXATIONS FOR PMI PROBLEMS}

In this section we expose the convex LMI relaxation methodology for non-convex PMI optimization problems. We first state formally the problem to be solved and introduce some notations. Then we briefly recall the main ideas for scalar polynomial optimization problems, in order to smoothly generalize them to matrix problems. Two small numerical examples illustrate the LMI relaxation procedure.

\section{A. PMI optimization}

Let $\mathcal{S}_{m}$ denote the space of real $m \times m$ symmetric matrices, and let the notation $A \succ 0$ (resp. $A \succeq 0$ ) stand for $A$ is positive definite (resp. positive semidefinite). Consider the optimization problem

$$
\begin{aligned}
& f^{\star}=\min f(x) \\
& \text { s.t. } \quad G(x) \succeq 0 \text {, }
\end{aligned}
$$

where $f$ is a real polynomial and $G: \mathbb{R}^{n} \rightarrow \mathcal{S}_{m}$, a polynomial mapping, i.e. each entry $G_{i j}(x)$ of the $m \times m$ symmetric matrix $G(x)$ is a polynomial in the indeterminate $x \in \mathbb{R}^{n}$. We will refer to problem (1) as a polynomial matrix inequality (PMI) optimization problem. Note that

- if $f$ and $G$ have degree ${ }^{1}$ one, then problem (1) is a convex linear matrix inequality (LMI) optimization problem;

- if $G$ has degree two with no square term, then problem (1) is a (generally non-convex) bilinear matrix inequality (BMI) optimization problem. By a slight abuse of terminology, BMI also sometimes refers to quadratic matrix inequalities.

\footnotetext{
${ }^{1}$ By degree of a polynomial matrix we mean the largest degree of all the scalar polynomial entries
}

This problem is a particular case of polynomial optimization problems considered in [19], [24] and the many references therein. Indeed, the matrix constraint $G(x) \succeq 0$ defines a semi-algebraic set $\mathcal{K} \subset \mathbb{R}^{n}$ that can be described explicitly in terms of $m$ scalar polynomial inequalities $g_{i}(x) \geq 0$, $i=1, \ldots, m$. The polynomials $g_{i}$ are obtained as follows. For every fixed $x \in \mathbb{R}^{n}$, let $t \mapsto p(t, x)=\operatorname{det}\left(t I_{m}-G(x)\right)$ be the characteristic polynomial of $G(x)$, and write $p$ in the form

$$
p(t, x)=t^{m}+\sum_{i=1}^{m}(-1)^{i} g_{i}(x) t^{m-i}, \quad t \in \mathbb{R} .
$$

Hence, as $t \mapsto p(t, x)$ has only real roots (because $G(x)$ is symmetric), we can use an extension (to nonnegative roots) of Descartes' rule of signs [1, p. 41] proved in [20]. That is, all the roots of $t \mapsto p(t, x)$ are nonnegative if and and only if $g_{i}(x) \geq 0$, for all $i=1, \ldots, m$. Therefore, in principle, the PMI problem (1) can be solved using recent LMI relaxation (also called semidefinite programming, or SDP relaxation) techniques developed in [19], and implemented in the software GloptiPoly [9]. In particular this approach allows to detect whether the global optimum is reached, and if so, to extract global minimizers, see [12].

However, the latter scalar representation of the PMI is perhaps not always appropriate, especially when $G(x)$ has high degree and/or dimension. Typically one polynomial $g_{i}(x)$ in (2) has high degree (for instance, in BMI problems polynomial $g_{0}(x)$ has potentially degree $2 m$ ). Recently, Hol and Scherer [14], [15] and Kojima [18] have tried to handle directly the matrix inequality constraint $G(x) \succeq 0$. Remarkably, they have derived a hierarchy of specific LMI relaxations, whose associated sequence of optimal values converges to the global optimum $f^{\star}$. However, and so far, only the convergence of the values has been obtained.

Our contribution is to complement these works by focusing on the dual of the LMI relaxations defined in [14], [15], [18] and briefly mentioned in [18]. In fact, a direct derivation of these LMI relaxations, in the spirit of the moment approach of [19], permits to retrieve the notions of moment and localizing matrices. Then, these LMI relaxations appear as genuine matrix analogues of the scalar LMI relaxations of [19]. A key feature of this dual approach is that we can apply verbatim the global optimality detection and global minimizer extraction procedures already available in the scalar case, and implemented in GloptiPoly.

\section{B. Moment and localizing matrices}

Let $\mathbb{R}\left[x_{1}, \ldots, x_{n}\right]$ denote the ring of real polynomials in the variables $x_{1}, \ldots, x_{n}$, also denoted by $\mathcal{P}$ as an $\mathbb{R}$-vector space, with associated canonical basis $b \in \mathcal{P}^{\infty}$, given by

$$
\begin{aligned}
x \mapsto b(x)= & {\left[\begin{array}{llllllll}
1 & x_{1} & x_{2} & \cdots & x_{n} & x_{1}^{2} & x_{1} x_{2} & \cdots \\
& \cdots & x_{1} x_{n} & x_{2} x_{3} & \cdots & x_{n}^{2} & x_{1}^{3} & \cdots
\end{array}\right]^{T} . }
\end{aligned}
$$

Let $y=\left\{y_{\alpha}\right\}_{\alpha \in \mathbb{N}^{n}}$ be a real-valued sequence indexed in the basis (3). A polynomial $p \in \mathcal{P}$ is also identified with its vector $\mathbf{p}=\left\{p_{\alpha}\right\}_{\alpha \in \mathbb{N}^{n}}$ of coefficients in the basis (3). For every 
$p \in \mathcal{P}$, the infinite vector $\mathbf{p}$ has only finitely many nontrivial entries. And so

$$
x \mapsto p(x)=\sum_{\alpha \in \mathbb{N}^{n}} p_{\alpha} x^{\alpha}=\langle\mathbf{p}, b(x)\rangle
$$

where $\langle A, B\rangle=\operatorname{trace}\left(A^{T} B\right)$ stands for the standard inner product of two matrices or vectors $A, B$ of compatible dimensions. For a fixed sequence $y=\left\{y_{\alpha}\right\}$ indexed in the basis (3), let $L_{y}: \mathcal{P} \mapsto \mathbb{R}$ be the linear mapping

$$
p \mapsto L_{y}(p)=\langle\mathbf{p}, y\rangle=\sum_{\alpha \in \mathbb{N}^{n}} p_{\alpha} y_{\alpha}
$$

Define the bilinear mapping $\langle., .\rangle_{y}: \mathcal{P} \times \mathcal{P} \mapsto \mathbb{R}$ by

$$
\langle p, q\rangle_{y}=L_{y}(p q)=\langle\mathbf{p}, M(y) \mathbf{q}\rangle
$$

for some infinite matrix $M(y)$, with rows and columns indexed in the basis $b$. With $\alpha, \beta \in \mathbb{N}^{n}$, the entry $(\alpha, \beta)$ of $M(y)$ is given by

$$
[M(y)]_{\alpha \beta}=L_{y}\left(\left[b(x) b(x)^{T}\right]_{\alpha \beta}\right)=y_{\alpha+\beta} .
$$

A sequence $y=\left\{y_{\alpha}\right\}$ is said to have a representing measure $\mu$ if

$$
y_{\alpha}=\int x^{\alpha} d \mu \quad \forall \alpha \in \mathbb{N}^{n}
$$

and in this case

$$
M(y)=\int b b^{T} d \mu=\int b(x) b(x)^{T} \mu(d x) .
$$

One can check that for any two polynomials $p, q \in \mathcal{P}$,

$$
\begin{aligned}
& L_{y}(p q)=\langle\mathbf{p}, M(y) \mathbf{q}\rangle=\int\left\langle\mathbf{p}, b b^{T} \mathbf{q}\right\rangle d \mu \\
& \quad=\int\langle\mathbf{p}, b(x)\rangle\langle b(x), \mathbf{q}\rangle \mu(d x)=\int p q d \mu .
\end{aligned}
$$

The infinite matrix $M(y)$, with rows and columns indexed in the basis $b$, is then called the moment matrix associated with the measure $\mu$. Now, denote by $b_{k}$ the canonical basis of the $\mathbb{R}$-vector subspace $\mathcal{P}_{k} \subset \mathcal{P}$ of real polynomials of degree at most $k$ (the finite truncation of $b$ in (3) which consists of monomials of degree at most $k$ ), and whose dimension is $s_{r}=\left(\begin{array}{c}n+r \\ r\end{array}\right)$. Then for all $p, q \in \mathcal{P}_{k}$

$$
L_{y}(p q)=\langle p, q\rangle_{y}=\left\langle\mathbf{p}, M_{k}(y) \mathbf{q}\right\rangle
$$

where $M_{k}(y)$ is the finite truncation of $M(y)$ with rows and columns indexed in the basis $b_{k}$. It immediately follows that if $y$ has a representing measure, then $M_{k}(y) \succeq 0$ for all $k=$ $0,1, \ldots$ because

$$
\langle p, p\rangle_{y}=\left\langle\mathbf{p}, M_{k}(y) \mathbf{p}\right\rangle=\int p^{2} d \mu \geq 0, \quad \forall p \in \mathcal{P}_{k} .
$$

Similarly, for a given polynomial $g \in \mathcal{P}$, let $\langle., .\rangle_{g y}: \mathcal{P} \times \mathcal{P} \mapsto$ $\mathbb{R}$ be the bilinear mapping

$$
(p, q) \mapsto\langle p, q\rangle_{g y}=L_{y}(g p q)=\langle\mathbf{p}, M(g y) \mathbf{q}\rangle
$$

where $M(g y)$ is called the localizing matrix associated with $y$ and $g \in \mathcal{P}$. With $\alpha, \beta, \gamma \in \mathbb{N}^{n}$ one can check that

$$
[M(g y)]_{\alpha \beta}=L_{y}\left(\left[g(x) b(x) b(x)^{T}\right]_{\alpha \beta}\right)=\sum_{\gamma} \mathbf{g}_{\gamma} y_{\alpha+\beta+\gamma}
$$

If $y$ has a representing measure $\mu$ with support contained in the closed set $\left\{x \in \mathbb{R}^{n} \mid g(x) \geq 0\right\}$, then

$$
\langle p, p\rangle_{g y}=\left\langle\mathbf{p}, M_{k}(g y) \mathbf{p}\right\rangle=\int g p^{2} d \mu \geq 0, \quad \forall p \in \mathcal{P}_{k}
$$

so that the truncated localizing matrix satisfies $M_{k}(g y) \succeq 0$, for all $k$.

\section{Scalar case}

In this section, we briefly recall the results of [19]. Consider the (generally non-convex) polynomial optimization problem

$$
\begin{array}{rl}
f^{\star}=\min _{x} & f(x) \\
\text { s.t. } & g_{i}(x) \geq 0, \quad i=1, \ldots, m
\end{array}
$$

where $f(x)$ and $g_{i}(x)$ are scalar real multivariate polynomials of the indeterminate $x \in \mathbb{R}^{n}$. Let

$$
\mathcal{K}=\left\{x \in \mathbb{R}^{n}: g_{i}(x) \geq 0, \quad i=1, \ldots, m\right\}
$$

denote the set of feasible solutions of (5), a semi-algebraic set of $\mathbb{R}^{n}$.

Problem (5) can be equivalently written as the moment optimization problem

$$
\begin{aligned}
f^{\star}=\min _{\mu} & \int_{\text {s.t. }} f(x) d \mu \\
\text { s } & \int_{\mathcal{K}} d \mu=1, \int_{\mathbb{R}-\mathcal{K}} d \mu=0 .
\end{aligned}
$$

In other words, we have replaced the (finite dimensional) nonconvex problem (5) with the convex (even linear !) optimization problem, but on an infinite dimensional space, namely, the space of measures on $\mathbb{R}^{n}$. At first glance, (6) seems to be just a rephrasing of the original problem (5), with no specific progress. However, we next use the fact that $f, g_{i}$ are all polynomials, in which case the formulation (6) can be further exploited.

Indeed, if $\mathcal{K}$ is compact, and under mild assumptions on the polynomials $g_{i}$ that define $\mathcal{K}$, one may define finitedimensional relaxations of the above problem (6) that involve finitely many moments of $\mu$, and whose sequence of optimal values converges to the desired global optimum $f^{*}$.

For $2 k \geq \max \left[\operatorname{deg} f, \max _{i} \operatorname{deg} g_{i}\right]$, consider the following semidefinite program

$$
\begin{array}{ll}
f^{(k)}=\min _{y} & L_{y}(f)\left(=\sum_{\alpha} f_{\alpha} y_{\alpha}\right) \\
\text { s.t. } & y_{0}=1 \\
& M_{k}(y) \succeq 0 \\
& M_{k-d_{i}}\left(g_{i} y\right) \succeq 0, \quad i=1, \ldots, m,
\end{array}
$$

where $y \in \mathbb{R}^{\left(s_{r}\right)}$, and $M_{k}(y) \succeq 0$ and $M_{k-d_{i}}\left(g_{i} y\right) \succeq 0$ are linear matrix inequality (LMI) constraints in $y$ corresponding to respective truncations of moment and localizing matrices, and where $2 d_{i}$ or $2 d_{i}-1$ is the degree of polynomial $g_{i}$ for $i=1, \ldots, m$. In other words, problem (7) is a convex LMI optimization problem. Obviously, the optimum $f^{(k)}$ is a lower bound on the global optimum $f^{\star}$ of the original problem, and $f^{(k)} \geq f^{\left(k^{\prime}\right)}$ whenever $k \geq k^{\prime}$. Problem (7) is referred to as the LMI relaxation of order $k$ of problem (5). 
Write $M_{k}(y)=\sum_{\alpha} B_{\alpha} y_{\alpha}$ and $M_{k-d_{i}}\left(g_{i} y\right)=\sum_{\alpha} C_{\alpha}^{i} y_{\alpha}$ for $i=1, \ldots, m$ and appropriate symmetric matrices $B_{\alpha}$ and $C_{\alpha}^{i}$. The dual of (7) is then the LMI problem

$$
\begin{aligned}
\lambda^{(k)}= & \\
\max _{\lambda, X, Z_{i}} & \lambda \\
\text { s.t. } & \left\langle B_{0}, X\right\rangle+\sum_{i=1}^{m}\left\langle C_{0}^{i}, Z_{i}\right\rangle=f_{0}-\lambda \\
& \left\langle B_{\alpha}, X\right\rangle+\sum_{i=1}^{m}\left\langle C_{\alpha}^{i}, Z_{i}\right\rangle=f_{\alpha}, \quad \forall 0 \neq|\alpha| \leq 2 k \\
& X \succeq 0, \quad Z_{i} \succeq 0, \quad i=1, \ldots, m .
\end{aligned}
$$

As shown in [19], the spectral decompositions of the positive semi-definite matrices $X, Z_{i}$ provide coefficient vectors of some associated sums of squares (s.o.s.) polynomials $p_{i}$, and the above LMI problem can be written as a polynomial s.o.s. problem

$$
\begin{aligned}
\lambda^{(k)}= & \\
\max _{\lambda, p_{i}} & \lambda \\
\text { s.t. } & f-\lambda=p_{0}+\sum_{i=1}^{m} p_{i} g_{i} \\
& p_{0}, \ldots, p_{m} \text { s.o.s. } \\
& \max \left[\operatorname{deg} p_{0}, \max _{i} \operatorname{deg} p_{i} g_{i}\right] \leq 2 k .
\end{aligned}
$$

Theorem 2.1: Assume that

- there exists a polynomial $p$ such that $p=p_{0}+\sum_{i} p_{i} g_{i}$ for some s.o.s. polynomials $p_{i}, i=0,1, \ldots, m$, and

- the level set $\left\{x \in \mathbb{R}^{n} \mid p \geq 0\right\}$ is compact.

Then, as $k \rightarrow \infty, f^{(k)} \uparrow f^{*}$ and $\lambda^{(k)} \uparrow f^{*}$ in LMI problems (7) and (8).

Proof: The proof can be sketched as follows, see [19] for details. Let $\epsilon \in \mathbb{R}>0$ be fixed arbitrary. The polynomial $f-f^{*}+\epsilon$ is strictly positive on $\mathcal{K}$. Then, by a representation theorem of Putinar [25]

$$
f-f^{*}+\epsilon=p_{0}+\sum_{i=1}^{m} p_{i} g_{i}
$$

for some s.o.s. polynomials $p_{i}, i=0,1, \ldots, m$. Let $2 k \geq$ $\max \left(\operatorname{deg} p_{0}, \max _{i} \operatorname{deg} p_{i} g_{i}\right)$. Then $\left(f^{*}-\epsilon, p_{0}, \ldots, p_{m}\right)$ is a feasible solution of (9) with value $\lambda=f^{*}-\epsilon$. By weak duality $\lambda^{(k)} \leq f^{(k)}$, and hence $f^{*}-\epsilon \leq \lambda^{(k)} \leq f^{(k)} \leq f^{*}$. As $\epsilon>0$ was arbitrary, the result follows.

The Matlab software GloptiPoly [9], released in 2002, builds up and solves the above LMI relaxations (7) of polynomial optimization problem (5). It was tested extensively on a set of benchmark engineering problems coming from continuous optimization, combinatorial optimization, polynomial systems of equations and control theory [9], [11]. In practice, it is observed that the global optimum is reached numerically (i.e. at given reasonable computational accuracy) at a relaxation order $k$ which is generally small (typically 1,2 or 3 ). Moreover, the relative gap $\left|f^{(k)}-f^{\star}\right|\left|f^{\star}\right|^{-1}$ is generally small for all $k$, meaning that the LMI relaxations generate good quality approximations.

Last but not least, a result of Curto and Fialkow [3] in the theory of moments can be exploited to detect whether the global optimum is reached numerically at a given relaxation order $k$, and to extract global minimizers $x^{*} \in \mathbb{R}^{n}$. All these tasks can be performed with standard numerical linear algebra (singular value decomposition, Cholesky factorization) and are implemented in GloptiPoly, see [12]. Thus, when some LMI relaxation is exact and the test of global optimality is passed, one also obtains one (or several) global minimizers, a highly desirable feature in most applications of interest.

\section{Matrix case}

To derive results in the matrix case, we proceed by close analogy with the scalar case described in the previous section. We now consider the PMI optimization problem (1), where $G: \mathbb{R}^{n} \rightarrow \mathcal{S}_{m}$ is a polynomial mapping in the indeterminate $x \in \mathbb{R}^{n}$. So, each entry $G_{i j}(x)=G_{j i}(x)$ of the matrix $G(x) \in$ $\mathcal{S}_{m}$ is a polynomial. Let

$$
\mathcal{K}:=\left\{x \in \mathrm{R}^{n}: G(x) \succeq 0\right\}
$$

denote the set of feasible solutions of (1), which is a semialgebraic set of $\mathbb{R}^{n}$.

For a polynomial mapping $P: \mathbb{R}^{n} \rightarrow \mathbb{R}^{m}$ of degree at most $k$, i.e. $P \in \mathcal{P}_{k}^{m}$, write $x \mapsto P(x)=\mathbf{P} b_{k}(x) \in \mathbb{R}^{m}$, for some $m \times s_{k}$ matrix $\mathbf{P}$, where $s_{k}$ is the dimension of the vector space $\mathcal{P}_{k}$ as defined previously. Also, the notation vecP denotes the vector obtained from the matrix $\mathbf{P}$ by stacking up its columns.

As in the scalar case, let $M_{k}(y)=\left\{y_{\alpha+\beta}\right\}_{\alpha, \beta}$, be the moment matrix of order $k$ (i.e. such that $|\alpha|,|\beta| \leq k$ ), associated with a sequence $y$. Similarly, we define the localizing matrix $M_{k}(G y)$, associated with a sequence $y$ and the polynomial matrix $x \mapsto G(x)$, as follows.

Writing $G(x)=\sum_{\gamma \in \mathbb{N}^{n}} G_{\gamma} x^{\gamma}$, for some finite family of real symmetric matrices $\left\{G_{\gamma}\right\}_{\gamma} \subset \mathcal{S}_{m}$, we also define the ( $m$-block) $s_{k}$-vector $G y$ by $(G y)_{\alpha}:=\sum_{\gamma} G_{\gamma} y_{\alpha+\gamma}$, for all $\alpha$ with $|\alpha| \leq k$. That is, each entry $(G y)_{\alpha},|\alpha| \leq k$, is a $m \times m$ matrix. Then, the localizing matrix $M_{k}(G y)$ has the block structure $\left\{(G y)_{\alpha+\beta}\right\}_{\alpha, \beta}$, with $|\alpha|,|\beta| \leq k$; equivalently, from its definition, $M_{k}(G y)$ is obtained from the moment matrix $M_{k}(y)$ by

$\left[M_{k}(G y)\right]_{\alpha \beta}=L_{y}\left(\left[\left(b_{k}(x) b_{k}(x)^{T} \otimes G(x)\right]_{\alpha \beta}\right), \quad|\alpha|,|\beta| \leq k\right.$,

where $\otimes$ stands for the Kronecker, or tensor product. In short and with abuse of notation, we can write

$$
M_{k}(G y)=L_{y}\left(b_{k}(x) b_{k}(x)^{T} \otimes G(x)\right)
$$

meaning that $L_{y}$ is applied entrywise to the polynomial matrix $x \mapsto b_{k}(x) b_{k}(x)^{T} \otimes G(x)$.

As in the scalar case, we come up with the following $k$ truncated linear problem

$$
\begin{array}{lll}
\mathbb{Q}_{k}: \quad f^{(k)}=\min _{y} & L_{y}(f)\left(=\sum_{\alpha} f_{\alpha} y_{\alpha}\right) \\
& \text { s.t. } & y_{0}=1 \\
& M_{k}(y) \succeq 0 \\
& M_{k-d}(G y) \succeq 0,
\end{array}
$$

where $M_{k}(y)$ and $M_{k-d}(G y)$ are the truncated moment and localizing matrices associated with $y$ and $G$.

Obviously, $f^{(k)} \leq f^{*}$ for all $k$ (i.e., $\mathbb{Q}_{k}$ is a relaxation of (1)) because if $x \in \mathbb{R}^{n}$ is feasible in (1) then $y:=b_{2 k}(x)$ is a feasible solution of (11). Indeed, $L_{y}(f)=f(x)$, and $M_{k}(y)=b_{k}(x) b_{k}(x)^{T} \succeq 0$. Finally, $M_{k-d}(y) \succeq 0$, because $M_{k-d}(y)$ is the tensor product of $G(x) \succeq 0$ and $M_{k-d}(y) \succeq 0$. 
Next, as in Hol and Scherer [14], [15], we say that a polynomial matrix $x \mapsto R(x) \in \mathcal{S}_{m}$ of dimension $m \times m$ and degree $2 k$ is s.o.s. if it can be written in the form

$$
x \mapsto R(x)=\sum_{j} Q_{j}(x) Q_{j}(x)^{T} \in \mathcal{S}_{m}
$$

for a family of polynomial vectors $Q_{j} \in \mathcal{P}_{k}^{m}$. Then, consider the polynomial s.o.s. problem

$$
\begin{aligned}
\lambda^{(k)}=\max _{\lambda, p_{0}, R} & \lambda \\
\text { s.t. } & f-\lambda=p_{0}+\langle R, G\rangle \\
& p_{0}, R \text { s.o.s. } \\
& \operatorname{deg} p_{0}, \operatorname{deg}\langle R, G\rangle \leq 2 k,
\end{aligned}
$$

the matrix analogue of (8). We can verify that

$$
\lambda^{(k)} \leq f^{(k)} .
$$

Indeed, let $\lambda, p_{0}, R$ be a feasible solution of (12), and let $y$ be a feasible solution of (11). Then from $f-\lambda=p_{0}+\langle R, G\rangle$, we obtain $L_{y}(f-\lambda)=L_{y}(f)-\lambda y_{0}=L_{y}(f)-\lambda=$ $L_{y}\left(p_{0}\right)+L_{y}(\langle R, G\rangle)$. We next prove that $L_{y}\left(p_{0}\right) \geq 0$ and $L_{y}(\langle R, G\rangle) \geq 0$, and so $L_{y}(f) \geq \lambda$, which in turn will imply (13).

As $p_{0}$ is s.o.s., say $p_{0}=\sum_{i=1}^{l} p_{0 l}^{2}$, for some family $\left\{p_{0 l}\right\} \subset \mathbb{R}[x]$, and using the linearity of $L_{y}$, we obtain $L_{y}\left(p_{0}\right)=\sum_{i=1}^{l} L_{y}\left(p_{0 l}^{2}\right)=\sum_{i=1}^{l}\left\langle p_{0 l}, M_{k}(y) p_{0 l}\right\rangle \geq 0$, because $M_{k}(y) \succeq 0$ (see (4)).

Similarly, write $x \mapsto R(x)=\sum_{j=1}^{l} Q_{j}(x) Q_{j}(x)^{T}$, with $Q_{j}(x)=\mathbf{Q}_{j} b_{k-d}(x)$ and where $\mathbf{Q}_{j} \in \mathbb{R}^{m \times s_{k-d}}$, for all $j=$ $1, \ldots, l$. Then, with $\mathbf{v}_{j}=\mathbf{v e c} \mathbf{Q}_{j}$ for all $j=1, \ldots, l$,

$$
\begin{aligned}
& \langle R(x), G(x)\rangle=\sum_{j=1}^{l}\left\langle\mathbf{Q}_{j} b_{k-d}(x) b_{k-d}(x)^{T} \mathbf{Q}_{j}^{T}, G(x)\right\rangle \\
& \quad=\sum_{j=1}^{l}\left\langle b_{k-d}(x) b_{k-d}(x)^{T} \otimes G(x), \mathbf{v}_{j} \mathbf{v}_{j}^{T}\right\rangle \\
& \quad=\sum_{j=1}^{l} \mathbf{v}_{j}^{T}\left[b_{k-d}(x) b_{k-d}(x)^{T} \otimes G(x)\right] \mathbf{v}_{j}
\end{aligned}
$$

and

$$
\begin{aligned}
& L_{y}(\langle R(x), G(x))\rangle \\
& \quad=\sum_{j=1}^{l} L_{y}\left(\mathbf{v}_{j}^{T}\left[b_{k-d}(x) b_{k-d}(x)^{T} \otimes G(x)\right] \mathbf{v}_{j}\right) \\
& \quad=\sum_{j=1}^{l}\left\langle\mathbf{v}_{j}, L_{y}\left(b_{k-d}(x) b_{k-d}(x)^{T} \otimes G(x)\right) \mathbf{v}_{j}\right\rangle \\
& \quad=\sum_{j=1}^{l}\left\langle\mathbf{v}_{j}, M_{k-d}(G y) \mathbf{v}_{j}\right\rangle \quad[\text { by (10)]. }
\end{aligned}
$$

Since $M_{k-d}(G y) \succeq 0$, it follows that $L_{y}(\langle R(x), G(x)\rangle) \geq 0$.

Therefore, we have proved that $L_{y}(f-\lambda) \geq 0$, i.e. $L_{y}(f) \geq$ $\lambda$ for any two solutions $y$ and $\lambda$ of (11) and (12) respectively, the desired result. In fact both LMI (11) and (12) are dual of each other.

We next use a result by $\mathrm{Hol}$ and Scherer [14], [15] and Kojima [18] to prove the following

Theorem 2.2: Assume that

- there exists a polynomial $p$ such that $p=p_{0}+\langle R, G\rangle$ for some s.o.s. polynomials $p_{0}$ and $R$, and

- the level set $\left\{x \in \mathbb{R}^{n} \mid p(x) \geq 0\right\}$ is compact.

Then, as $k \rightarrow \infty, f^{(k)} \uparrow f^{*}$ and $\lambda^{(k)} \uparrow f^{*}$ in LMI relaxations (11) and (12).

Proof: We already have $f^{(k)} \leq f^{*}$ for all $k$, and from (13), $\lambda^{(k)} \leq f^{(k)} \leq f^{*}$. Next, under the assumption of the theorem, Hol and Scherer [14], [15] and Kojima [18] have proved that $\lambda^{(k)}$ in (12), satisfies $\lambda^{(k)} \uparrow f^{*}$ as $k \rightarrow \infty$. From what precedes, the result follows.
Remark 2.3: The assumptions of Theorem 2.2 are not very restrictive. For instance, suppose that one knows an a priori bound $\rho$ on the Euclidean norm $\left\|x^{*}\right\|$ of a global minimizer $x^{*}$. Then, one introduces the new BMI constraint $\tilde{G}(x)=$ $\operatorname{diag}\left\{G(x), \rho^{2}-\|x\|^{2}\right\} \succeq 0$ and the feasibility set $\tilde{\mathcal{K}}=\{x \in$ $\left.\mathbb{R}^{n}: \tilde{G}(x) \succeq 0\right\}$ for which both assumptions are satisfied. Indeed, let $e \in \mathbb{R}^{m+1}$ be such that $e_{j}=\delta_{j, m+1}$ for all $j=$ $1, \ldots, m+1$. Then, the polynomial $x \mapsto p(x)=\rho^{2}-\|x\|^{2}$ can be written as $p=\left\langle e e^{T}, \tilde{G}\right\rangle$ and the level set $\left\{x \in \mathbb{R}^{n}: p \geq 0\right\}$ is compact.

We now prove a result that permits to detect whether the LMI $\mathbb{Q}_{k}$ provides the optimal value $f^{*}$, and if so, global minimizers as well. This is important because it will permit to use the extraction procedure already described in [12], and obtain global minimizers, exactly in the same manner as in the scalar case. We strongly use an important theorem of Curto and Fialkow [3] on (positive) flat extensions of moment matrices.

Theorem 2.4: Suppose that an optimal solution $y^{*}$ of the LMI $\mathbb{Q}_{k}$ in (11) satisfies

$$
s=\operatorname{rank} M_{k}\left(y^{*}\right)=\operatorname{rank} M_{k-d}\left(y^{*}\right) .
$$

Then $y^{*}$ is the vector of moments (up to order $2 k$ ) of an $s$ atomic probability measure $\mu^{*}$ with support contained in the set $\mathcal{K}$. That is, there are $s$ distinct points $\left\{x_{j}\right\}_{j=1}^{s} \subset \mathcal{K}$ such that

$$
\mu^{*}=\sum_{j=1}^{s} \gamma_{j} \delta_{x_{j}}, \quad \sum_{j=1}^{s} \gamma_{j}=1, \quad \gamma_{j}>0, \quad j=1, \ldots, s
$$

and $G\left(x_{j}\right) \succeq 0$, where $\delta_{x}$ denotes the Dirac measure at $x \in$ $\mathbb{R}^{n}$. Therefore $f^{k}=f^{*}$ and $x_{1}, \ldots, x_{s}$ are global minimizers.

Proof: From (14), $M_{k}\left(y^{*}\right)$ is a flat extension of $M_{k-d}\left(y^{*}\right)$, that is, $M_{k}\left(y^{*}\right) \succeq 0, M_{k-d}\left(y^{*}\right) \succeq 0$ and $\operatorname{rank} M_{k-d}\left(y^{*}\right)=\operatorname{rank} M_{k}\left(y^{*}\right)$. Therefore, by the flat extension theorem, $y^{*}$ is the vector of moments (up to order $2 k$ ) of some $s$-atomic probability measure $\mu^{*}$ on $\mathbb{R}^{n}$, see [3] or [21, Theor. 1.3]. That is, there are $s$ distinct points $\left\{x_{j}\right\}_{j=1}^{s} \subset \mathbb{R}^{n}$ such that (15) is satisfied.

Next, let $\left\{\lambda_{j}\right\}_{j=1}^{s}$ be an arbitrary set of nonzero eigenvalues of the matrices $\left\{G\left(x_{j}\right)\right\}_{j=1}^{s} \subset \mathbb{R}^{m \times m}$, with associated set $\left\{u_{j}\right\}_{j=1}^{s} \subset \mathbb{R}^{m}$ of (normalized) eigenvectors. That is, $G\left(x_{j}\right) u_{j}=\lambda_{j} u_{j}$, with $\lambda_{j} \neq 0$, for all $j=1, \ldots, s$. As $s=$ $\operatorname{rank} M_{k-d}\left(y^{*}\right)$, then there exist $s$ interpolation polynomials $\left\{g_{i}\right\}_{i=1}^{s} \subset \mathbb{R}[x]$ at points $\left\{x_{j}\right\}_{j=1}^{s}$, of degree at most $k-d$, i.e., $g_{i}\left(x_{j}\right)=\delta_{i j}$ for $i, j=1, \ldots, s$, where $\delta_{i j}$ is the Kronecker symbol; see [21, Lemma 2.7].

Then for every $j=1, \ldots, s$, let $H_{j} \in \mathcal{P}_{k-d}^{m}$ be the polynomial vector $\mathbb{R}^{n} \rightarrow \mathbb{R}^{m}$,

$$
x \mapsto H_{j}(x)=g_{j}(x) u_{j}, \quad j=1, \ldots, s .
$$

Then observe that $H_{j}\left(x_{k}\right)=\delta_{j k} u_{k}$ for all $j, k=1, \ldots, s$. In addition, by the feasibility of $y^{*}$ in the LMI $\mathbb{Q}_{k}$, for every $j=1, \ldots, s$,

$$
\begin{aligned}
& \left\langle\mathbf{v e c H}_{j}, M_{k-d}\left(G y^{*}\right) \mathbf{v e c H}_{j}\right\rangle \\
& \quad=\int\left\langle H_{j}(x), G(x) H_{j}(x)\right\rangle d \mu^{*} \\
& \quad=\sum_{l=1}^{s} \gamma_{l}\left\langle H_{j}\left(x_{l}\right), G\left(x_{l}\right) H_{j}\left(x_{l}\right)\right\rangle \\
& \quad=\gamma_{j}\left\langle H_{j}\left(x_{j}\right), G\left(x_{j}\right) H_{j}\left(x_{j}\right)\right\rangle \\
& \quad=\gamma_{j} \lambda_{j} \geq 0 .
\end{aligned}
$$


Therefore, as $\gamma_{j}>0$, it follows that $\lambda_{j} \geq 0$ for all $j=$ $1, \ldots, s$. As the set of nonzero eigenvalues $\left\{\lambda_{j}\right\}_{j=1}^{s}$ was arbitrary, it thus follows that all eigenvalues of $G\left(x_{j}\right)$ are nonnegative, i.e., $G\left(x_{j}\right) \succeq 0$, for all $j=1, \ldots, s$. And so, the probability measure $\mu^{*}$ is supported on the set $\mathcal{K}$, the desired result.

Using the condition (14) in Theorem 2.4, we can now extract global minimizers $x^{*} \in \mathbb{R}^{n}$, exactly along the same lines described in [12]. It is illustrated in the examples below.

\section{E. First example}

Consider the $2 \times 2$ quadratic matrix inequality in 2 variables

$$
\begin{array}{rl}
f^{\star}= & \\
\min _{x} & f(x)=-x_{1}^{2}-x_{2}^{2} \\
\text { s.t. } & G(x)=\left[\begin{array}{cc}
1-4 x_{1} x_{2} & x_{1} \\
x_{1} & 4-x_{1}^{2}-x_{2}^{2}
\end{array}\right] \succeq 0 .
\end{array}
$$

The non convex bounded feasible set $\mathcal{K}=\{x \mid G(x) \succeq 0\}$ is represented in Figure 1. There are two global optima $x=$ $[0, \pm 2]$, both with optimal value $f^{\star}=-4$.

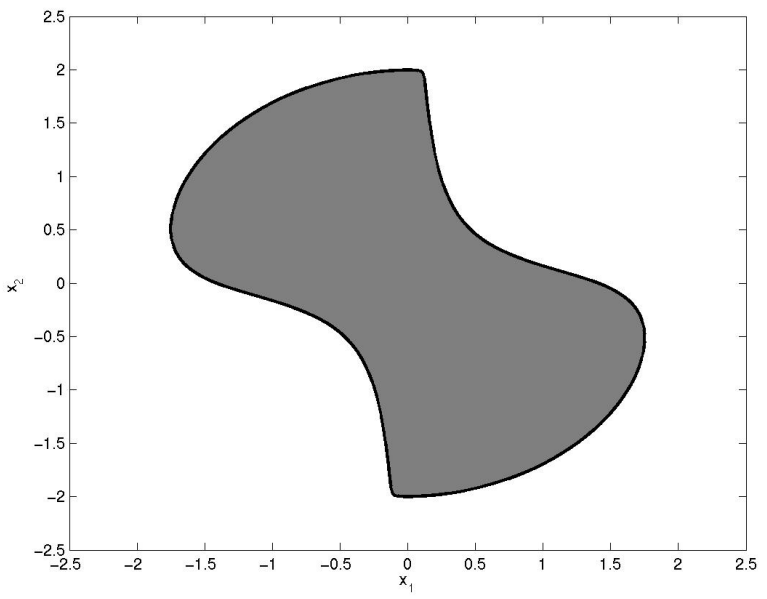

Fig. 1. Example II-E. Non-convex PMI feasible set.

1) Scalarizing: First, we translate the PMI optimization problem (16) into a scalar polynomial optimization problem, see Section II-A.

The quadratic matrix inequality $G(x) \succeq 0$ is feasible if and only if the characteristic polynomial $t \mapsto p(t, x)=\operatorname{det}(t I-$ $G(x))=t^{2}-\left(5-4 x_{1} x_{2}-x_{1}^{2}-x_{2}^{2}\right) t+\left(4-16 x_{1} x_{2}-2 x_{1}^{2}-x_{2}^{2}+\right.$ $\left.4 x_{1}^{3} x_{2}+4 x_{1} x_{2}^{3}\right)$ has only non-negative real roots. According to [20], this is the case if and only if the coefficients of $p(t, x)$ have alternating signs, i.e. if and only if

$$
\begin{aligned}
& g_{1}(x)=5-4 x_{1} x_{2}-x_{1}^{2}-x_{2}^{2} \geq 0 \\
& g_{2}(x)=4-16 x_{1} x_{2}-2 x_{1}^{2}-x_{2}^{2}+4 x_{1}^{3} x_{2}+4 x_{1} x_{2}^{3} \geq 0 .
\end{aligned}
$$

Our PMI optimization problem (16) is then equivalent to the scalar polynomial optimization problem

$$
\begin{array}{ll}
f^{\star}=\min _{x} & f(x) \\
\text { s.t. } & g_{1}(x) \geq 0 \\
& g_{2}(x) \geq 0 .
\end{array}
$$

\begin{tabular}{c|c|c|c|c}
$\begin{array}{c}\text { LMI } \\
\text { relax. } \\
\text { order } k\end{array}$ & $\begin{array}{c}\text { Lower } \\
\text { bound } \\
f^{(k)}\end{array}$ & $\begin{array}{c}\text { Ranks of } \\
\text { moment } \\
\text { matrices }\end{array}$ & $\begin{array}{c}\text { Number } \\
\text { of LMI } \\
\text { variables }\end{array}$ & $\begin{array}{c}\text { Size } \\
\text { of LMI } \\
\text { constraints }\end{array}$ \\
\hline 2 & -4.8382 & 3,4 & 14 & $6+3+1$ \\
3 & -4.2423 & $3,5,7$ & 27 & $10+6+3$ \\
4 & -4.0947 & $3,6,10,12$ & 44 & $15+10+6$ \\
5 & -4.0353 & $3,6,10,14,16$ & 65 & $21+15+10$ \\
6 & -4.0062 & $3,6,10,15,21,28$ & 90 & $28+21+15$ \\
7 & -4.0000 & $2,2,2,2,2,2,2$ & 119 & $36+28+21$
\end{tabular}

\section{TABLE I}

EXAMPLE II-E. APPLYING GLOPTIPOLY ON THE SCALARIZED PMI

Applying GloptiPoly on this problem, using the LMI solver SeDuMi with default tunings, we get the results summarized in Table I. We report there the LMI relaxation order $k$, the computed lower bounds $f^{(k)}$ on the global optimum, the ranks of partitions of successive moment matrices, as well as the number of scalar variables and the size of the LMI constraints. Note that we have to start with the LMI relaxation of order $k=2$ since the scalarized problem features a polynomial $g_{2}$ of degree $2 k=4$.

The global optimum is reached at the 7th LMI relaxation: the moment matrix has rank two and the two global optima are extracted using the algorithm described in [12].

2) Keeping the matrix structure: Now we apply the LMI relaxations described in section II-D. They keep the matrix structure of the PMI.

The first LMI relaxation of the PMI optimization problem (16) is given by

$$
\begin{aligned}
& f^{(1)}=\min -y_{20}-y_{02} \\
& \text { s.t. } M_{1}(y)=\left[\begin{array}{c|ll}
1 & & \\
\hline y_{10} & y_{20} & \\
y_{01} & y_{11} & y_{02}
\end{array}\right] \succeq 0 \\
& M_{0}(G y)=\left[\begin{array}{cc}
1-4 y_{11} & \\
y_{10} & 4-y_{20}-y_{02}
\end{array}\right] \succeq 0,
\end{aligned}
$$

where symmetric upper triangular entries are omitted.

The second LMI relaxation is given by

$$
\begin{aligned}
& f^{(2)}=\min -y_{20}-y_{02} \\
& \text { s.t. } \quad M_{2}(y)=\left[\begin{array}{c|cc|ccc}
1 & & & & & \\
\hline y_{10} & y_{20} & & & & \\
y_{01} & y_{11} & y_{02} & & & \\
\hline y_{20} & y_{30} & y_{21} & y_{40} & & \\
y_{11} & y_{21} & y_{12} & y_{31} & y_{22} & \\
y_{02} & y_{12} & y_{03} & y_{22} & y_{13} & y_{04}
\end{array}\right] \succeq 0 \\
& M_{1}(G y)=\left[\begin{array}{c|cc}
M_{00}(G y) & & \\
\hline M_{10}(G y) & M_{20}(G y) & \\
M_{01}(G y) & M_{11}(G y) & M_{02}(G y)
\end{array}\right] \succeq 0
\end{aligned}
$$




\begin{tabular}{c|c|c|c|c}
$\begin{array}{c}\text { LMI } \\
\text { relax. } \\
\text { order } k\end{array}$ & $\begin{array}{c}\text { Lower } \\
\text { bound } \\
f^{(k)}\end{array}$ & $\begin{array}{c}\text { Ranks of } \\
\text { moment } \\
\text { matrices }\end{array}$ & $\begin{array}{c}\text { Number } \\
\text { of LMI } \\
\text { variables }\end{array}$ & $\begin{array}{c}\text { Size } \\
\text { of LMI } \\
\text { constraints }\end{array}$ \\
\hline 1 & -4.0000 & 3 & 5 & $3+2$ \\
2 & -4.0000 & 2,2 & 14 & $6+6$
\end{tabular}

TABLE II

EXAMPle II-E. Solving THE LMI RELAXATIONS OF THE PMI.

where

$$
\begin{aligned}
M_{00}(G y) & =\left[\begin{array}{cc}
1-4 y_{11} & \\
y_{10} & 4-y_{20}-y_{02}
\end{array}\right] \\
M_{10}(G y) & =\left[\begin{array}{cc}
y_{10}-4 y_{21} & \\
y_{20} & 4 y_{10}-y_{30}-y_{12}
\end{array}\right] \\
M_{01}(G y) & =\left[\begin{array}{cc}
y_{01}-4 y_{12} & \\
y_{11} & 4 y_{01}-y_{21}-y_{03}
\end{array}\right] \\
M_{20}(G y) & =\left[\begin{array}{cc}
y_{20}-4 y_{31} & \\
y_{30} & 4 y_{20}-y_{40}-y_{22}
\end{array}\right] \\
M_{11}(G y) & =\left[\begin{array}{cc}
y_{11}-4 y_{22} & \\
y_{21} & 4 y_{11}-y_{31}-y_{13}
\end{array}\right] \\
M_{02}(G y) & =\left[\begin{array}{cc}
y_{02}-4 y_{13} \\
y_{12} & 4 y_{02}-y_{22}-y_{04}
\end{array}\right] .
\end{aligned}
$$

Solving these two LMI relaxations, we get the results summarized in Table II. We see that, in contrast with the scalarization technique, the global optimum is reached already at the first LMI relaxation, at a very moderate cost. We only have to resort to the second LMI relaxation in order to obtain a numerical certificate of global optimality and to extract the two solutions, also at a very moderate cost when compared with the scalarization technique. Remarkably, we have only used moment variables $y_{\alpha}$ of order at most 4 , in contrast to 14 , in the scalar case.

For illustration, we briefly recall the extraction procedure described in [12]. At the optimum, the moment matrix of the second LMI relaxation, rounded to three significant digits, is given by

$$
M_{2}\left(y^{*}\right)=\left[\begin{array}{c|cc|ccc}
1.00 & & & & & \\
\hline 0.00 & 0.00 & & & & \\
0.00 & 0.00 & 4.00 & & & \\
\hline 0.00 & 0.00 & 0.00 & 0.00 & & \\
0.00 & 0.00 & 0.00 & 0.00 & 0.00 & \\
4.00 & 0.00 & 0.00 & 0.00 & 0.00 & 16.0
\end{array}\right] .
$$

A Cholesky factorization, obtained via e.g. the Schur decomposition or the singular value decomposition, is given by

$M_{2}\left(y^{*}\right)=\left[\begin{array}{cc}1.00 & 0.00 \\ \hline 0.00 & 0.00 \\ 0.00 & 1.00 \\ \hline 0.00 & 0.00 \\ 0.00 & 0.00 \\ 4.00 & 0.00\end{array}\right]\left[\begin{array}{ll}1.00 & 0.00 \\ 0.00 & 4.00\end{array}\right]\left[\begin{array}{ll}1.00 & 0.00 \\ 0.00 & 0.00 \\ 0.00 & 1.00 \\ \hline 0.00 & 0.00 \\ 0.00 & 0.00 \\ 4.00 & 0.00\end{array}\right]$

From this rank-two Cholesky factor in echelon form, it follows from [12] that global optimizers satisfy the polynomial system

\begin{tabular}{c|c|c|c|c}
$\begin{array}{c}\text { LMI } \\
\text { relax. } \\
\text { order } k\end{array}$ & $\begin{array}{c}\text { Lower } \\
\text { bound } \\
f^{(k)}\end{array}$ & $\begin{array}{c}\text { Ranks of } \\
\text { moment } \\
\text { matrices }\end{array}$ & $\begin{array}{c}\text { Number } \\
\text { of LMI } \\
\text { variables }\end{array}$ & $\begin{array}{c}\text { Size } \\
\text { of LMI } \\
\text { constraints }\end{array}$ \\
\hline 2 & -1.8926 & 2,2 & 14 & $6+3+1$ \\
3 & -1.8926 & $2,2,2$ & 27 & $10+6+3$
\end{tabular}

TABLE III

EXAMPLE II-F. APPLYING GLOPTIPOLY ON THE SCALARIZED PMI.

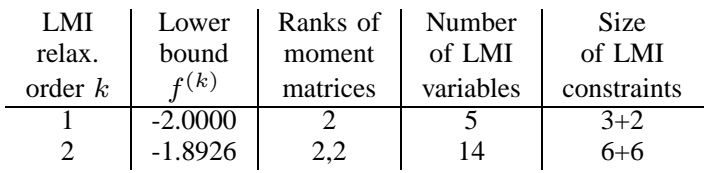

TABLE IV

EXAMPLE II-F. SOLVING THE LMI RELAXATIONS BY KEEPING THE MATRIX STRUCTURE OF THE PMI.

of equations

$$
\begin{aligned}
1 & =1 \\
x_{1} & =0 \\
x_{2} & =x_{2} \\
x_{1}^{2} & =0 \\
x_{1} x_{2} & =0 \\
x_{2}^{2} & =4
\end{aligned}
$$

whose right hand-side can be expressed in the polynomial basis $\left(1, x_{2}\right)$. Note that these equations come from the polynomials lying in the kernel of the moment matrix. As explained in [12], this kind of polynomial system can be solved via eigenvalue extraction. Here it is straightforward to conclude that the two global optimizers are $x=\left[\begin{array}{ll}0 & \pm 2\end{array}\right]$.

\section{F. Second example}

Now change the objective function in example II-E to

$$
f(x)=x_{1} x_{2} .
$$

1) Scalarizing: Solving the scalarized problem with GloptiPoly, we get the results reported in Table III, showing that the global optimum is now reached at the second LMI relaxation, and certified at the third LMI relaxation. The two extracted solutions are $x= \pm[-1.3383,1.4142]$, with optimal value $f^{*}=-1.8926$. Here, we have used moment variables $y_{\alpha}$ of order at most 6 .

2) Keeping the matrix structure: Solving the LMI relaxations of the PMI by keeping the matrix structure, we obtain the results summarized in Table IV. The global optimum is reached and certified at the second LMI relaxation. Here the advantage of keeping the matrix structure of the PMI is less apparent, but we still have only used moment variables of order at most 4 , in contrast to 6 in the scalar case.

\section{APPLICATION TO STATIC OUTPUT FEEDBACK DESIGN}

In this section we apply the LMI relaxation methodology of section II to solve PMI optimization problems arising from static output feedback (SOF) design problems. After recalling 
the SOF problem statement and its standard BMI state-space formulation, we propose an alternative PMI polynomial formulation. Then we illustrate the relevance of the LMI relaxation mechanism on non-trivial PMI problems arising from SOF problems.

\section{A. SOF design}

Consider the linear system

$$
\begin{aligned}
\dot{x} & =A x+B u \\
y & =C x
\end{aligned}
$$

of order $n$ with $m$ inputs and $p$ outputs, that we want to stabilize by static output feedback

$$
u=K y \text {. }
$$

In other words, given matrices $A \in \mathbb{R}^{n \times n}, B \in \mathbb{R}^{n \times m}$, $C \in \mathbb{R}^{p \times n}$, we want to find matrix $K \in \mathbb{R}^{m \times p}$ such that the eigenvalues of closed-loop matrix $A+B K C$ all belong to a region

$$
\mathcal{D}=\{s \in \mathbb{C}: a+b(s+\bar{s})+c s \bar{s}<0\}
$$

of the complex plane, where $a, b, c \in \mathbb{R}$ are given scalars and the bar denotes the complex conjugate. Typical choices are $a=c=0, b=1$ for the left half-plane (continuous-time stability) and $c=-a=1, b=0$ for the unit disk (discretetime stability).

Problem SOF: Given matrices $A, B, C$, find matrix $K$ such that eigenvalues of matrix $A+B K C$ all belong to given stability region $\mathcal{D}$.

\section{B. State-space BMI formulation}

Following a standard state-space approach [27], the SOF problem can be formulated as the BMI

$$
(A+B K C) T P+(A+B K C) P \prec 0, \quad P=P^{T} \succ 0
$$

in decision variables $K$ and $P$ where $\prec 0$ and $\succ 0$ stand for positive and negative definite, respectively. We see that SOF matrix $K$ (the actual problem unknown) contains $m p$ scalar entries, whereas Lyapunov matrix $P$ (instrumental to ensuring stability) contains $n(n+1) / 2$ scalar entries. When $n$ is significantly larger than $m p$, the important number of resulting Lyapunov variables may be prohibitive.

\section{PMI formulation}

In this section we propose an alternative PMI formulation of the SOF problem featuring entries of matrix $K$ only. In order to get rid of the Lyapunov variables, we focus on a polynomial formulation of the SOF problem, applying the Hermite stability criterion on the closed-loop characteristic polynomial, in the spirit of [8].
1) Characteristic polynomial: Let $\kappa \in \mathbb{R}^{m p}$ be the vector obtained by stacking the columns of matrix $K$. Define

$$
q(s, \kappa)=\operatorname{det}(s I-A-B K C)=\sum_{i=0}^{n} q_{i}(\kappa) s^{i}
$$

as the characteristic polynomial of matrix $A+B K C$. Coefficients of increasing powers of indeterminate $s$ in polynomial $q(s, \kappa)$ are multivariate polynomials in $\kappa$, i.e.

$$
q_{i}(\kappa)=\sum_{\alpha} q_{i_{\alpha}} \kappa^{\alpha}
$$

where $\alpha \in \mathbb{N}^{m p}$ describes all monomial powers.

2) Hermite stability criterion: The roots of polynomial $q(s, \kappa)$ belong to stability region $\mathcal{D}$ if and only if

$$
H(\kappa)=\sum_{i=0}^{n} \sum_{j=0}^{n} q_{i}(\kappa) q_{j}(\kappa) H_{i j} \succ 0
$$

where $H(\kappa)=H^{T}(\kappa) \in \mathbb{R}^{n \times n}$ is the Hermite matrix of $q(s, \kappa)$. Coefficients $H_{i j}=H_{i j}^{T} \in \mathbb{R}^{n \times n}$ depend on the stability region $\mathcal{D}$ only, see [10].

3) Polynomial matrix inequality: Hermite matrix $H(\kappa)$ depends polynomially on vector $\kappa$, hence the equivalent notation

$$
H(\kappa)=\sum_{\alpha} H_{\alpha} \kappa^{\alpha} \succ 0
$$

where matrices $H_{\alpha}=H_{\alpha}^{T} \in \mathbb{R}^{n \times n}$ are obtained by combining matrices $H_{i j}$, and $\alpha \in \mathbb{N}^{m p}$ describes all monomial powers.

Lemma 3.1: Problem SOF is solved if and only if vector $\kappa$ solves the PMI (17).

\section{Numerical aspects}

In [13], we discuss various numerical aspects regarding the derivation of PMI (17). For conciseness, they are only briefly mentioned here and not reported in full detail:

- Computing the characteristic polynomial: to build up polynomial $q(s, \kappa)$ we need to evaluate coefficients $q_{i_{\alpha}}$ of the determinant of matrix $s I-A-B K C$. We proceed numerically by interpolation: coefficients of $q(s, \kappa)$ are determined by solving a linear system of equation built on a perfectly conditioned truncated multivariate Vandermonde matrix;

- Building up the Hermite matrix: coefficients $H_{i j}$ depend only on the stability region $\mathcal{D}$. They are computed by solving a simple linear system of equations, as shown in [10]. In the case of continuous-time stability, the Hermite matrix can be split down into two blocks of approximate half size;

- Strict feasibility: to solve the strict PMI feasibility problem (17), we can solve the non-strict problem

$$
H(\kappa) \succeq \lambda I
$$

trying e.g. to maximize scalar $\lambda>0$. In practice however the feasibility set of PMI (17) can be unbounded in some directions and $\lambda$ can grow unreasonably large. In our experiments we set $\lambda$ to some small positive constant value; 
- Minimizing the trace of the moment matrix: as noticed in [12] for such problems, in order to improve convergence of the hierarchy of LMI relaxations, it is recommended to minimize the trace of the moment matrix $M_{k}(y)$. Existence of a scalar $\gamma>0$ such that

$$
\text { trace } M_{k}(y) \leq \gamma
$$

ensures boundedness of all the moments $y_{\alpha}$, and thus, feasibility of the relaxations.

\section{E. Numerical experiments}

In this section we report numerical experiments showing that the methodology developed in section II can indeed prove useful for solving non-trivial SOF problems formulated in this polynomial setting. The problems are extracted from the publicly available benchmark collection $\mathrm{COMPl}_{e} \mathrm{ib}_{\text {[22]. These }}$ problems are formulated in continuous-time (region $\mathcal{D}$ is the left half-plane, $a=c=0, b=1$ ). LMI problems were built with the YALMIP Matlab interface [23] and solved with SeDuMi [29] with default tunings. When testing ranks of moment matrices, we use a relative gap threshold of $10^{-4}$ between successive singular values. Numerical data are rounded to 5 digits.

1) Example AC8: A model of a modern transport airplane with $n=9$ states, $m=1$ input and $p=5$ outputs. The statespace BMI formulation of Section III-B would introduce 45 scalar Lyapunov variables in addition to the 5 feedback gain entries. Scalarization as in Section II-A would result in a set of 9 scalar polynomial constraints of degree up to 18 in 5 variables. Therefore, the first LMI relaxation in the hierarchy (7) would involve $\left(\begin{array}{c}23 \\ 5\end{array}\right)=33649$ variables.

By keeping the matrix structure, solving the first LMI relaxation (24 moment variables, LMI size 5+4+6) returns a moment matrix $M_{1}$ whose 4 largest singular values are 1.0000 , $5.6595 \cdot 10^{-6}, 2.3851 \cdot 10^{-7}$ and $2.2305 \cdot 10^{-7}$. So matrix $M_{1}$ has numerical rank one, the global optimum is reached, and factorizing $M_{1}$ yields the stabilizing feedback matrix

$$
\begin{aligned}
& K=\left[\begin{array}{lll}
3.6275 \cdot 10^{-6} & -3.8577 \cdot 10^{-4} & \ldots
\end{array}\right. \\
& \left.-1.0121 \cdot 10^{-5} \quad 1.7389 \cdot 10^{-3} \quad 2.0960 \cdot 10^{-4}\right] \text {. }
\end{aligned}
$$

Observe that one obtains the global optimum at a relaxation that involves moments of order up to 2 only.

2) Example REA3: A model of a nuclear reactor with $n=$ 12 states, $m=1$ input and $p=3$ outputs. The state-space BMI formulation would introduce 78 scalar Lyapunov variables in addition to the 5 feedback gain entries. Scalarization would result in a set of 12 scalar polynomial constraints of degree up to 24 in 3 variables. Therefore, the first LMI relaxation in the hierarchy (7) would involve $\left(\begin{array}{c}27 \\ 3\end{array}\right)=2925$ variables.

Solving the first LMI relaxation (10 variables, LMI size $6+6+4)$ returns a moment matrix $M_{1}$ whose 4 singular values are $6326.0,1.0000,2.1075 \cdot 10^{-7}$ and $1.3116 \cdot 10^{-6}$. Matrix $M_{1}$ has numerical rank two.

Solving the second LMI relaxation (35 variables, LMI size $24+24+10$ ) returns a moment matrix $M_{1}$ with singular values $6327.0,2.4620 \cdot 10^{-3}, 1.9798 \cdot 10^{-3}, 3.9060 \cdot 10^{-6}$ and a moment matrix $M_{2}$ whose 4 largest singular values are 4.0025 .
$10^{7}, 21.092,15.397$ and 4.6680 . We consider that both $M_{1}$ and $M_{2}$ have rank one so that the global optimum is reached. Factorizing $M_{1}$ yields the stabilizing feedback matrix

$$
K=\left[\begin{array}{lll}
-1.1037 \cdot 10^{-7} & -0.15120 & -79.536
\end{array}\right] .
$$

One obtains the global optimum at a relaxation that involves moments of order up to 4 only.

3) Example HE1: A model of the longitudinal motion of a helicopter, with $n=4$ states, $m=2$ inputs and $p=1$ output.

Solving the first LMI relaxation (6 variables, LMI size $2+2+3$ ) returns a moment matrix $M_{1}$ whose 3 singular values are $1.0076,2.6562 \cdot 10^{-2}, 2.1971 \cdot 10^{-9}$ so matrix $M_{1}$ has numerical rank two.

Solving the second LMI relaxation (15 variables, LMI size $6+6+6)$ returns a moment matrix $M_{1}$ with singular values $1.0085,6.4009 \cdot 10^{-2}, 6.9224 \cdot 10^{-10}$ and a moment matrix $M_{2}$ whose 4 largest singular values are $1.0128,8.0720 \cdot 10^{-2}$, $1.7875 \cdot 10^{-2}, 8.0773 \cdot 10^{-10}$. So matrix $M_{1}$ has numerical rank two, whereas matrix $M_{2}$ has numerical rank three, and we cannot conclude.

Solving the third LMI relaxation (28 variables, LMI size $12+12+10)$ we obtain a moment matrix $M_{1}$ with singular values $1.1404,9.8176 \cdot 10^{-10}, 4.5344 \cdot 10^{-11}$, a moment matrix $M_{2}$ with 4 largest singular values $1.1583,1.1052 \cdot 10^{-9}$, $8.0379 \cdot 10^{-11}, 6.0171 \cdot 10^{-11}$, and a moment matrix $M_{3}$ with 4 largest singular values $1.1605,1.1716 \cdot 10^{-9}, 3.8334 \cdot 10^{-10}$, $7.2405 \cdot 10^{-11}$. All these moment matrices have numerical rank one, so the global optimum is reached. Factorizing $M_{1}$ yields the stabilizing feedback matrix

$$
K=\left[\begin{array}{ll}
-0.11972 & 0.35500
\end{array}\right] .
$$

One obtains the global optimum at a relaxation that involves moments of order up to 6 only. The global optimum, together with the non-convex set of stabilizing SOF gains, are represented in Figure 2.

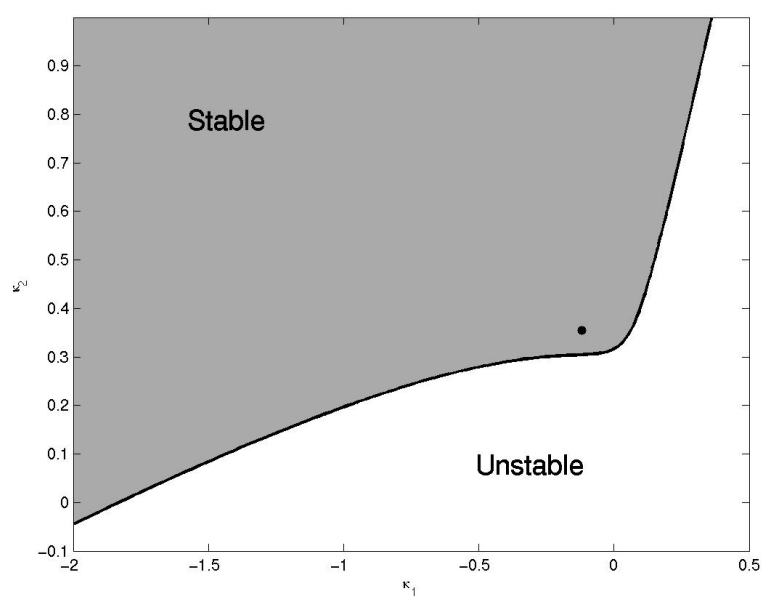

Fig. 2. Example HE1. Non-convex set of stabilizing SOF gains (gray zone) and global optimum (black dot). 


\section{CONCLUSION}

We have proposed a methodology to solve, in a systematic way, non-convex polynomial matrix inequalities (PMI) problems. Based on a moment interpretation of recent results on sum-of-squares decompositions of positive polynomial matrices, a hierarchy of convex linear matrix inequality (LMI) relaxations is built up, with a guarantee of convergence to the global optimum of the original non-convex PMI problem. When finite convergence occurs (as observed in practice), results from the theory of moments allows to detect global optimality and extract global optimizers with the help of existing numerical linear algebra algorithms. It is planned to incorporate PMI constraints into the next release of the GloptiPoly software [9].

The methodology is then applied to solve non-trivial static output feedback (SOF) problems formulated as PMI problems. Since the number of variables as well as the number of constraints both grow relatively fast when building up the hierarchy of successive LMI relaxations, it is important to reduce the number of variables in the SOF PMI problem as much as possible. Our approach for solving SOF problems allows this by focusing on an algebraic, or polynomial formulation: namely, the Hermite stability criterion is applied on the closed-loop characteristic polynomial, resulting in PMI SOF stabilizability conditions involving feedback matrix gain entries only, without additional Lyapunov variables.

One may argue that every PMI problem can be transformed into an equivalent scalar polynomial optimization problem by an application of Descartes' rule of signs as in Section IIA. Therefore, theoretically, one may solve a PMI problem by solving the hierarchy of LMI relaxations defined in [19], and implemented in the software GloptiPoly [9]. However, notice that at least one polynomial in the scalar representation of the PMI has high degree, which induces LMI relaxations of size too large for the present status of SDP solvers, see Examples REA 3 and A8 of Section III-E. In contrast, the approach developed in the present paper takes explicitly into account the matrix structure of the PMI problems and the designer has a better control on the size growth of the successive LMI relaxations in the hierarchy.

As far as control applications are concerned, the PMI formulation must be extended to cope with $H_{2}$ or $H_{\infty}$ performance criteria. The key issue is to formulate these criteria algebraically, without using state-space arguments. Similarly as for the SOF design problem, all the instrumental Lyapunov variables must be removed in order to derive a PMI formulation directly in the controller parameters.

Several numerical aspects of PMI problems deserve to be studied in further detail. In our opinion, the field of numerical analysis for polynomials (monovariate, multivariate, scalar or matrix) is still mostly unexplored [28]. There is a crucial need for reliable numerical software dealing with polynomials and polynomial inequalities. Other potentially interesting research topics include reducing the number of constraints in a PMI (removing redundant semi-algebraic constraints), detecting convexity (some PMI SOF problems are convex) or exploiting the structure of the LMI relaxations in interior point schemes.

\section{ACKNOWLEDGMENTS}

This work benefited from several stimulating discussions with Camile Hol and Carsten Scherer. This work was partly supported by grant No. NT05-3-41612 of the French National Research Agency (ANR), grant No. 102/02/0709 of the Grant Agency of the Czech Republic, and project No. ME 698/2003 of the Ministry of Education of the Czech Republic.

\section{REFERENCES}

[1] S. Basu, R. Pollack, M. F. Roy. Algorithms in Real Algebraic Geometry, Springer, Berlin, 2003

[2] S. Boyd, L. El Ghaoui, E. Feron, V. Balakrishnan. Linear matrix inequalities in system and control theory. SIAM, Philadelphia, 1994.

[3] R.E. Curto, L.A. Fialkow. Solution of the truncated complex moment problem with flat data. Mem. Amer. Math. Soc. 119(568), 1996.

[4] H. Fujioka, K. Hoshijima. Bounds for the BMI eigenvalue problem. Trans. Soc. Instr. Control Engrs., 33(7):616-621, 1997.

[5] M. Fukuda, M. Kojima. Branch-and-cut algorithms for the bilinear matrix inequality eigenvalue problem. Comput. Optim. Appli., 19(1):79105,2001

[6] K. C. Goh, M. G. Safonov, G. P. Papavassilopoulos. Global optimization for the biaffine matrix inequality problem. J. Global Opt., 7(4):365-380, 1995.

[7] K. C. Goh, M. G. Safonov, J. H. Ly. Robust synthesis via bilinear matrix inequality. Int. J. Robust and Nonlinear Control, 6(9/10):1079_ 1095, 1996.

[8] D. Henrion, S. Tarbouriech, M. Šebek. Rank-one LMI approach to simultaneous stabilization of linear systems. Syst. Control Letters, 38(2):79-89, 1999.

[9] D. Henrion, J. B. Lasserre. GloptiPoly: global optimization over polynomials with Matlab and SeDuMi. ACM Trans. Math. Soft. 29(2):165194, 2003.

[10] D. Henrion, D. Peaucelle, D. Arzelier, M. Šebek. Ellipsoidal approximation of the stability domain of a polynomial. IEEE Trans. Autom. Control, 48(12):2255-2259, 2003.

[11] D. Henrion, J. B. Lasserre. Solving nonconvex optimization problems. IEEE Control Syst. Mag., 24(3):72-83, 2004

[12] D. Henrion, J. B. Lasserre. Detecting global optimality and extracting solutions in GloptiPoly. Contributed chapter in D. Henrion, A. Garulli (Editors). Positive polynomials in control. LNCIS 312, Springer Verlag, Berlin, 2005.

[13] D. Henrion, J. Löfberg, M. Kočvara, M. Stingl. Solving static output feedback problems with PENBMI. Proc. joint IEEE Conf. Decision and Control and European Control Conf., Sevilla, Spain, Dec. 2005.

[14] C. W. J. Hol, C. W. Scherer. Sum of squares relaxations for polynomial semidefinite programming. Proc. Symp. on Mathematical Theory of Networks and Systems (MTNS), Leuven, Belgium, July 2004.

[15] C. W. J. Hol, C. W. Scherer. A sum of squares approach to fixed-order $H_{\infty}$ synthesis. Contributed chapter in D. Henrion, A. Garulli (Editors). Positive polynomials in control. LNCIS, Springer Verlag, Berlin, 2005.

[16] M. Kočvara, M. Stingl. PENNON - a code for convex nonlinear and semidefinite programming. Opt. Methods and Software, 18(3):317-333, 2003.

[17] M. Kočvara, M. Stingl. PENBMI, Version 2.0, 2004. See www. penopt.com for a free developer version.

[18] M. Kojima. Sums of squares relaxations of polynomial semidefinite programs. Research report B-397, Dept. Math. Comput. Sc., Tokyo Inst. Tech, Japan, Nov. 2003.

[19] J. B. Lasserre. Global optimization with polynomials and the problem of moments. SIAM J. Opt. 11(3):796-817, 2001.

[20] J. B. Lasserre. A unified criterion for positive definiteness and semidefiniteness. Research Report 05283, LAAS-CNRS Toulouse, France, May 2005.

[21] M. Laurent. Revisiting two theorems of Curto and Fialkow on moment matrices. Proc. American Math. Society, 133(10):2965-2976, 2005.

[22] F. Leibfritz. COMPleib:: constrained matrix optimization problem library - a collection of test examples for nonlinear semidefinite programs, control system design and related problems. Research report, Department of Mathematics, University of Trier, Germany, 2003. See www. compleib.de.

[23] J. Löfberg. YALMIP: a toolbox for modeling and optimization in Matlab. Proc. of the IEEE Computer-Aided Control System Design Conference, Taipei, Taiwan, Sept. 2004. See control.ee.ethz.ch/ joloef. 
[24] P. A. Parrilo. Semidefinite programming relaxations for semialgebraic problems. Math. Prog. Ser. B, 96(2):293-320, 2003.

[25] M. Putinar. Positive polynomials on compact semi-algebraic sets, Indiana Univ. Math. J. 42:969-984, 1993.

[26] C. W. Scherer, S. Weiland. Course on LMIs in Control, Lecture Notes at Delft University of Technology and Eindhoven University of Technology, The Netherlands, 2002.

[27] R. E. Skelton, T. Iwasaki, K. M. Grigoriadis. A unified algebraic approach to linear control design. Taylor and Francis, New York, 1997.

[28] H. J. Stetter. Numerical polynomial algebra. SIAM, Philadelphia, 2004.

[29] J. F. Sturm. Using SeDuMi 1.02, a Matlab toolbox for optimization over symmetric cones. Opt. Methods and Software, 11-12:625-653, 1999. 